\title{
On the Non-primitive Variables Formulations for the Incompressible Euler Equations
}

\section{Shaaban Abdallah}

Department of Aerospace Engineering and Engineering Mechanics University of Cincinnati, Cincinnati, Ohio, USA

Corresponding author: Shaaban Abdallah, Department of Aerospace Engineering and Engineering Mechanics University of Cincinnati, Cincinnati, Ohio, USA, Tel: 513-556-3321; E-mail: Shaaban.abdallah@uc.edu

Received date: May 30, 2017, Accepted date: May 31, 2017, Published date: June 9, 2017

Copyright: (c) 2017 Abdallah S. This is an open-access article distributed under the terms of the Creative Commons Attribution License, which permits unrestricted use, distribution, and reproduction in any medium, provided the original author and source are credited.

\section{Editorial}

The Euler equations for inviscid incompressble flow are hyperbolicelliptic equations in terms of primitive variables velocity and pressure. Numerical solutions for the Euler equations can be generally classified under primitive variables, and non-primitive variables formulations. The non-primitive variables formulation defines new dependent variables to resolve numerical difficulties in solutions of the primitive variables equations. For example, using vorticity as a dependent variable several techniques have been developed suitable for numerical solutions such as the stream function-vorticity, the velocity-vorticity, etc.

This article addresses two non-primitive variables formulations namely the Clebsch [1], and the Impulse density [2-7] formulations. The objective is to compare the two formulations to identify similarities, advantages/ or disadvantages relative to each other, and relative to the vorticity based formulations. The Clebsch formulation decomposes the velocity vector into a rotational (non- zero Curl) and a non-rotational (gradient of a potential function) velocity components. The rotational component is modeled by two functions that are governed by Lagrangian equations derived from the momentum equation. By substitution into the continuity equation, a Poisson equation for the potential function is obtained. On the other hand, the Impulse density formulations define a new time dependent variable that consists of two components, the first is a divergence free (the velocity vector itself), and the second component is a curl free (gradient of a potential function). Upon substitution into the continuity equation, similar to the Clebsch formulation, a Poisons equation is obtained for the potential function. And, the momentum equation transforms into a hyperbolic first order equation with arbitrary gauge function. The arbitrary choice of the gauge function has advantages, over vorticity based formulations, in designing efficient/or accurate numerical methods for solutions of the Euler equations.

The Euler Equations for Incompressible flow:

$$
\begin{aligned}
& \partial \mathrm{tu}+\mathrm{u} \cdot \mathrm{u}=-\mathrm{p} \\
& \cdot \mathrm{u}=0
\end{aligned}
$$

Where $\mathrm{u}$ is the velocity vector and $\mathrm{p}$ is the static pressure.

The Clebsch formulation:

The velocity vector is decomposed into a rotational velocity component $\mathrm{Q}$ and a potential component $\varphi$,

$$
\mathrm{u}=\mathrm{Q}-\varphi
$$

The rotational component $\mathrm{Q}$ is molded by two functions $\lambda$ and $\mu$

$$
\mathrm{Q}=\lambda \mu
$$

Taking the curl of equation (3), we obtain:

$$
\omega=\lambda \times \mu
$$

The momentum equation reduces to

$$
\begin{aligned}
& \operatorname{Dt}(\lambda)=0 \\
& \operatorname{Dt}(\mu)=0
\end{aligned}
$$

Where

$$
D t=\partial t+u \text {. }
$$

Substituting equation (3) into equation (2), one obtains:
$2 \varphi=\cdot \mathrm{Q}$
(9) The Impulse density formulation:
$\mathrm{u}=\mathrm{q}-\varphi$

Where $\mathrm{q}$ is the Impulse density. By substituting equation (10) into equation (1), and choosing the geometric gauge function $[1,2,5]$, one obtains:

$$
\partial \mathrm{t}(\mathrm{q})+\mathrm{u} \cdot \mathrm{q}=-(\mathrm{u}) \mathrm{T} \mathrm{q}
$$

The potential equation (9) is also applicable here with $\mathrm{Q}$ being replaced by q.

By comparing equations (10) and (3), the Impulse density vector $\mathrm{q}$ corresponds to the rotational velocity component $\mathrm{Q}$, and the potential component in equation (10) corresponds to the potential component in equation (3). In references [2] and [3], it is shown that the vortex lines are orthogonal to the material surface represented by the Impulse density vector q. Similarly, in the Clebsch formulation, the vortex lines are obliviously orthogonal to the rotational velocity component, see equations (4) and (5).

In conclusion, interesting similarities between the Clebsch and the Impulse density formulations are pointed out. Both formulations represent the velocity vector by two components governed by hyperbolic and elliptic equations. The difference between the two formulations is the arbitrary gauge function of the Impulse density formulation that can be employed for designing efficient or accurate numerical methods. An important feature that is unique to both the Clebsch and the Impulse density relative to the vorticity based formulations is that the velocity components are time dependent even for steady state solutions. Finally, the number of dependent variables in the Clebsch formulation is three governed by equations (6), (7), and (9), for two- and three- dimensions. And the number of dependent variables in the Impulse density formulation changes from three in two-dimensions to four in three- dimensions, equations (9) and (11). 
Citation: Abdallah S (2017) On the Non-primitive Variables Formulations for the Incompressible Euler Equations. Global J Technol Optim 8: e111.

Page 2 of 2

\section{References}

1. Clebsch A (1859) Concerning the integration of hydrodynamic equations.. J Reine Angew Math 56: 1-10.

2. Weinan E, Liu JG (1997) Finite difference schemes for incompressible flows in the velocity-impulse density formulation. J Comput Phys 130: 67.

3. Russo G, Smereka P (1999) Impulse formulation of the Euler equations: general properties and numerical methods. J Fluid Mech 391: 189-209.

4. Oseledets VI (1989) On a new way of writing the Navier-Stokes equation: The Hamiltonian formalism. Russ Math Surveys 44: 210.
5. Smereka P (1996) A Vlasov description of the Euler equation. Nonlinearity 9: 1361

6. Sagdeev RZ, Moiseev SS, Tur AV, Yanovsky VV (1986) Problems of the theory of string turbulence and topological solitons. In Nonlinear Phenomena in Plasma Physics and Hydrodynamics, Mir Publishers, Moscow.

7. Tur AV, Yanonsky VV (1993) Invariants in dissipationless hydrodynamic media. J Fluid Mech 248: 67-106. 